\title{
Preface - Special Issue Dedicated to Professor Scott E. Denmark in Honor of his $65^{\text {th }}$ Birthday
}

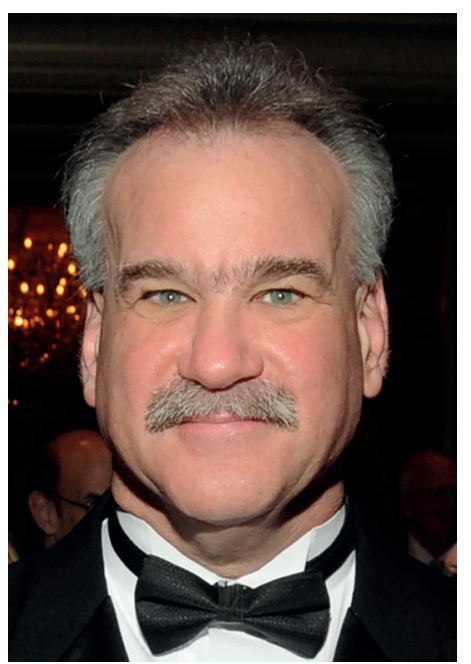

This SYNTHESIS special issue is dedicated to Professor Scott E. Denmark in honor of his 65th Birthday. Its genesis followed from discussions with Professor Michael Harmata, one of Scott Denmark's first graduate students at the University of Illinois at Urbana-Champaign. Below we provide a general statement that captures Scott Denmark's immense contributions to organic chemistry, followed by two personal statements from each, as we were fortunate to have been members of his research group.

Professor Denmark has been highly influential in the discipline and internationally recognized as a leader in the development of novel reactivity concepts as well as catalysts and reagents that lead to powerful synthetic methods for organic chemistry. His distinctive contributions to a remarkably diverse group of reaction classes are characterized by high originality, unusual thoroughness, and extraordinary demanding experimental standards. The breadth of his research accomplishments reflects an intellectual virtuosity in the application of chemical principles to important synthetic and mechanistic problems. A compelling impulse to understand the fundamentals of structure and reactivity is clearly the motivating force behind all of his research endeavors in synthetic methodology. The Denmark approach to organic synthesis is exceptional for its thorough documentation of generality, careful attention to stereochemical detail, and extensive investigation of mechanistic underpinnings. Thieme is thus is delighted to assemble a Special Issue that captures cutting edge chemistry to highlight the first-class science that characterizes Scott Denmark's own transformative contributions to the science of organic chemistry.
As editor for Special Issues in Synthesis, I had the good fortune to take a class in advanced organic chemistry taught by Professor Denmark and to have worked in his group as an undergraduate researcher. Through my interactions with Professor Denmark I was initiated to the wonders of organic chemistry; it is a fascination that persists to this day. Moreover, I have always considered myself fortunate to count Professor Denmark as a lifelong mentor and friend, who has profoundly influenced my life. Happy Birthday Scott!

Erick M. Carreira, SYNTHESIS Editor for Special Issues and Special Topics, and Professor at the Organic Chemistry Laboratory, ETH Zürich

This past year Professor Scott E. Denmark turned 65. Although it is hard to tell, it is somewhat of a scary thought, but only because it means I am getting older too. I met Scott in 1980 at the University of Illinois. We were both in our first year, he a professor and me a graduate student. I had no intention of joining his group, as I did not know he existed. But when he gave his spiel on his research plans to the new graduate students, I was blown away. What he could do with a blackboard and a piece of white chalk to stir the passions of those who love chemistry was utterly amazing.

At that time, Scott did not have much of a paper trail. He was not well published, but apparently came highly recommended by a Swiss named Albert Eschenmoser. I was too naïve to know much of anything about that aspect of science at that time. I just knew Scott was a ball of fire, with ideas that were incredible. A few others and I others joined the group, and we are all better scientists for it.

The rest of the story is well known by all. Scott took the chemistry world by storm, winning many accolades, and demonstrating a commitment and integrity to the science that is the gold standard for those in our field. He is both a synthetic chemist and a physical organic chemist, dedicated to methodology and total synthesis, and driven to understand the very essence of how reactions work. His accomplishments were rewarded most recently by election to the National Academy of Science, no doubt due to the spirit of scientific excellence and integrity he espouses; I was thrilled when I heard the news.

A few of his former co-workers put together a small tribute to Scott on the occasion of his birthday, as represented by the papers contained herein. It has been a pleasure to know him for what is now approaching 40 years. Scott, may you have many more years of enjoying the exploration of the wonders of chemistry. Happy Birthday!

Michael Harmata, Norman Rabjohn Distinguished Professor of Chemistry, University of Missouri 